\title{
APPLICATION OF JACOBI POLYNOMIALS TO THE APPROXIMATE SOLUTION OF A SINGULAR INTEGRAL EQUATION WITH CAUCHY KERNEL ON THE REAL HALF-LINE
}

\author{
D. PYLAK ${ }^{1}$
}

\begin{abstract}
In this paper, exact solution of the characteristic equation with Cauchy kernel on the real half-line is presented. Next, Jacobi polynomials are used to derive approximate solutions of this equation. Moreover, estimations of errors of the approximated solutions are presented and proved.
\end{abstract}

2000 Mathematics Subject Classification: 65R20, 45L10, 45E05.

Keywords: Jacobi polynomials, Cauchy-type kernels, singular equations, approximate solutions.

\section{Introduction}

We consider the characteristic equation

$$
a \varphi(x)+\frac{1}{\pi i} \int_{0}^{+\infty} \frac{b \varphi(\sigma)}{\sigma-x} d \sigma=f(x), \quad x \in(0,+\infty),
$$

where $a, b$ are given complex numbers satisfying the conditions $a^{2}-b^{2} \neq 0$ and $b \neq 0, f(x)$ is the given complex-valued Hölder continuous function and $\varphi(x)$ is the unknown function.

We say, that a function $f(x), x \in[0,+\infty)$, belongs to the Hölder class if it satisfies the inequality

$$
\left|f\left(x_{1}\right)-f\left(x_{2}\right)\right| \leqslant A_{1}\left|x_{1}-x_{2}\right|^{\gamma}, \quad 0<\gamma \leqslant 1, \quad \forall x_{1}, x_{2} \in[0, c], \quad A_{1}=\text { const },
$$

on each interval $[0, c], c>0$, and the condition

$$
|f(x)-f(\infty)| \leqslant A_{2} /|x|^{\gamma}, \quad A_{2}=\text { const },
$$

is satisfied as $x \rightarrow \infty$.

If the integration contour is a smooth closed or open curve of finite length, the theory of equations of the form (1.1) has been developed comprehensively in $[1,2,6,11]$. Moreover, the equation

$$
\frac{1}{\pi i} \int_{0}^{+\infty} \frac{\varphi(\sigma)}{\sigma-x} d \sigma=f(x), \quad x>0
$$

which is a special case of $(1.1)$, was considered in $[1,8,10]$.

\footnotetext{
${ }^{1}$ Department of Mathematics, The John Paul II Catholic University of Lublin, Al. Raclawickie 14, 20-950 Lublin, Poland. E-mail: dorotab@kul.lublin.pl
} 
In the present paper, we find explicit formulas for the solution of (1.1). We also find conditions for the unique solvability of (1.1). Moreover, we present numerical schemes for (1.1) based on Jacobi polynomials and estimate the order of accuracy of the approximate solution.

\section{The characteristic singular integral equation with a Cauchy kernel on the real half-line}

We apply the transformation [9] of the form

$$
\frac{1}{\sigma-x}=\frac{x+1}{\sigma+1} \frac{1}{\sigma-x}+\frac{1}{\sigma+1}
$$

to the kernel of (1.1) and rewrite the equation in the form

$a \varphi(x)+\frac{1}{\pi i} \int_{0}^{+\infty} \frac{x+1}{\sigma+1} \frac{b}{\sigma-x} \varphi(\sigma) d \sigma+\frac{1}{\pi i} \int_{0}^{+\infty} \frac{b}{\sigma+1} \varphi(\sigma) d \sigma=f(x), \quad x \in(0,+\infty), \quad a^{2}-b^{2} \neq 0$.

By setting $x=(1+t) /(1-t), \sigma=(1+\tau) /(1-\tau)$ and introducing the notation

$$
\psi(t)=\varphi\left(\frac{1+t}{1-t}\right), \quad g(t)=f\left(\frac{1+t}{1-t}\right)
$$

we reduce (2.1) to the form

$$
\begin{gathered}
a \psi(t)+\frac{1}{\pi i} \int_{-1}^{1} b \frac{\psi(\tau)}{\tau-t} d \tau-\frac{1}{\pi i} \int_{-1}^{1} b \frac{\psi(\tau)}{\tau-1} d \tau=g(t), \quad t \in(-1,1), \\
\lim _{t \rightarrow 1^{-0}} \psi(t)=0, \quad \lim _{t \rightarrow 1^{-0}} g(t)=0 .
\end{gathered}
$$

Let $X(z)$ be a canonical function for the linear conjugation problem

$$
X^{+}(t)=\frac{a-b}{a+b} X^{-}(t), \quad-1<t<1,
$$

and

$$
Z(t)=(a+b) X^{+}(t)=(a-b) X^{-}(t), \quad-1<t<1 .
$$

If the solution $\psi(t)$ belongs to $h(1)$ (i.e., the class of Hölder continuous functions on $(-1,1)$, bounded in the neighborhood of the point $z=1$ and admitting an integrable singularity at the point $z=-1[6])$, then, for $0<\theta<\pi$, we have

$$
X(z)=\left(\frac{z-1}{z+1}\right)^{\omega_{1}+i \omega_{2}}, \quad z \notin(-1,1)
$$

and for $-\pi<\theta<0$

$$
X(z)=\left(\frac{z-1}{z+1}\right)^{\omega_{1}+i \omega_{2}} \frac{z-1}{z+1}, \quad z \notin(-1,1),
$$

where $G=(a-b) /(a+b) \theta=\arg G, \omega_{1}=(2 \pi)^{-1} \theta$ and $\omega_{2}=-(2 \pi)^{-1} \ln |G|$. 
Hence, in the first case we have

$$
Z(t)=\sqrt{a^{2}-b^{2}} p(t),
$$

where

$$
p(t)=(1-t)^{\alpha}(1+t)^{\beta}, \quad \alpha=\omega_{1}+i \omega_{2}, \quad \beta=-\omega_{1}-i \omega_{2},
$$

and in the second case

$$
Z(t)=(-1) \sqrt{a^{2}-b^{2}} p(t)
$$

where

$$
p(t)=(1-t)^{\alpha}(1+t)^{\beta}, \quad \alpha=1+\omega_{1}+i \omega_{2}, \quad \beta=-1-\omega_{1}-i \omega_{2} .
$$

The index of the characteristic operator in the class $h(1)$ is equal to $0(\kappa=-(\alpha+\beta)=0)$.

If the solution $\psi(t)$ is sought in the class $h(-1,1)$ (i. e., the class of Hölder continuous functions on $(-1,1)$, bounded in the neighbourhoods of points $z= \pm 1[6])$, then the fundamental solution $X(z)$ has the form

$$
X(z)=\left(\frac{z-1}{z+1}\right)^{\omega_{1}+i \omega_{2}}(z+1), \quad 0<\theta<\pi
$$

and

$$
X(z)=\left(\frac{z-1}{z+1}\right)^{\omega_{1}+i \omega_{2}}(z-1), \quad-\pi<\theta<0
$$

respectively. Thus, for $0<\theta<\pi$, we have

$$
Z(t)=\sqrt{a^{2}-b^{2}}(1-t)^{\alpha}(1+t)^{\beta}, \quad \alpha=\omega_{1}+i \omega_{2}, \quad \beta=1-\omega_{1}-i \omega_{2},
$$

and, for $-\pi<\theta<0$, we have

$$
Z(t)=-\sqrt{a^{2}-b^{2}}(1-t)^{\alpha}(1+t)^{\beta}, \quad \alpha=1+\omega_{1}+i \omega_{2}, \quad \beta=-\omega_{1}-i \omega_{2} .
$$

In this case, the index $\kappa$ is equal to $-1(\kappa=-(\alpha+\beta)=-1)$.

Passing in (2.2) to the new unknown function $u(t)$ by the rule

$$
\psi(t)=\frac{Z(t)}{a^{2}-b^{2}} u(t)
$$

and introducing the notation $A=a /\left(a^{2}-b^{2}\right), B=b /\left(a^{2}-b^{2}\right)$, we reduce (2.2) to the form $A Z(t) u(t)+\frac{1}{\pi i} \int_{-1}^{1} B Z(\tau) \frac{u(\tau)}{\tau-t} d \tau-\frac{1}{\pi i} \int_{-1}^{1} B Z(\tau) \frac{u(\tau)}{\tau-1} d \tau=g(t), \quad t \in(-1,1), \quad g(1)=0$.

Setting

$$
\frac{1}{\pi i} \int_{-1}^{1} B Z(\tau) \frac{u(\tau)}{\tau-1} d \tau=\gamma_{0}
$$

and solving (2.15) in the class $h(1)$, we obtain

$$
u(t)=a Z^{-1}(t) g(t)-\frac{1}{\pi i} \int_{-1}^{1} b Z^{-1}(\tau) \frac{g(\tau)}{\tau-t} d \tau+\gamma_{0}
$$


Hence by substituting (2.17) into (2.15) we can show that the function $(2.17)$, where $\gamma_{0}$ is an arbitrary constant, is the general solution of (2.15) [9].

The constant $\gamma_{0}$ on the right-hand side in expression (2.17) is uniquely determined if (2.15) is supplemented by the condition

$$
\frac{1}{\pi i} \int_{-1}^{1} B Z(\tau) \frac{u(\tau)}{\tau-1} d \tau=A_{0}^{*}
$$

equivalent to (2.16). Substituting function (2.17) for $u(t)$ into this relation, we obtain $\gamma_{0}=A_{0}^{*}$.

Let us introduce function classes that will be used during the solution of (1.1). We write $\varphi(x) \in h(\infty), x>0$, if $\varphi(x)$ belongs to the class of functions that are Hölder continuous on $[\epsilon,+\infty), \epsilon>0$, vanish at infinity $\left(\lim _{x \rightarrow+\infty} \varphi(x)=0\right)$, and can have an integrable singularity in the neighbourhood of $x=0$. Further, we write $\varphi(x) \in h(0, \infty), x>0$, if it belongs to the class of bounded Hölder functions on $(0,+\infty)$ such that $\lim _{x \rightarrow+\infty} \varphi(x)=0$.

We have thereby proved the following theorem.

Theorem 2.1. Let a complex valued function $f(x) \in h(0, \infty)$ be given on $[0,+\infty)$. Then the general solution of Eq. (1.1) in the class $h(\infty)$ has the form

$$
\begin{gathered}
\varphi(x)=\frac{a}{a^{2}-b^{2}} f(x)-\frac{x^{\beta}}{a^{2}-b^{2}} \frac{1}{\pi i} \int_{0}^{+\infty} \frac{b f(\sigma)}{\sigma^{\beta}(\sigma-x)} d \sigma+ \\
\frac{x^{\beta}}{a^{2}-b^{2}} \frac{1}{\pi i} \int_{0}^{+\infty} \frac{b f(\sigma)}{\sigma^{\beta}(\sigma+1)} d \sigma+\frac{\gamma_{0}}{\sqrt{a^{2}-b^{2}} x^{-\beta}}, \quad x \in(0,+\infty),
\end{gathered}
$$

where $\operatorname{Re} \beta<0,0<\theta<\pi$, and $\gamma_{0}$ is an arbitrary constant. Additionally, if the solution $\varphi(x)$ satisfies the condition

$$
\frac{1}{\pi i} \int_{0}^{+\infty} \frac{b \varphi(\sigma)}{\sigma+1} d \sigma=A_{0}^{*}
$$

where $A_{0}^{*}$ is an arbitrary number, then the unique solution of problem (1.1) is given by formula (2.19) with $\gamma_{0}=A_{0}^{*}$.

Now we will look for the solution $u(t)$ of $(2.15)$ in $h(-1,1)$. From $[2,6,9]$ it follows that (2.15) is solvable if and only if $(\pi i)^{-1} \int_{-1}^{1} b Z^{-1}(t)\left[g(t)+A_{0}^{*}\right] d t=0$, where $A_{0}^{*}$ is given by (2.18). Since it follows from [11] that $(\pi i)^{-1} \int_{-1}^{1} b Z^{-1}(t) d t=\operatorname{Res}_{z=\infty}(X(z))^{-1}=-1$, we have

$$
A_{0}^{*}=\frac{1}{\pi i} \int_{-1}^{1} B Z(\tau) \frac{u(\tau)}{\tau-1} d \tau=\frac{1}{\pi i} \int_{-1}^{1} b Z^{-1}(\tau) g(\tau) d \tau
$$

Therefore, (2.15) can be rewritten in the form

$$
A Z(t) u(t)+\frac{1}{\pi i} \int_{-1}^{1} \frac{B Z(\tau) u(\tau)}{\tau-t} d \tau=g(t)+\frac{1}{\pi i} \int_{-1}^{1} b Z^{-1}(\tau) g(\tau) d \tau, \quad t \in(-1,1)
$$


If the necessary and sufficient condition (2.21) is satisfied, then in view of $[2,6,9]$ the solution of this equation is given by the formula

$$
\begin{gathered}
u(t)=a Z^{-1}(t)\left(g(t)+A_{0}^{*}\right)-\frac{1}{\pi i} \int_{-1}^{1} b Z^{-1}(\tau) \frac{\left(g(\tau)+A_{0}^{*}\right)}{\tau-t} d \tau= \\
a Z^{-1}(t) g(t)-\frac{1}{\pi i} \int_{-1}^{1} b Z^{-1}(\tau) \frac{g(\tau)}{\tau-t} d \tau, \quad t \in(-1,1) .
\end{gathered}
$$

This finishes the proof of the following theorem.

Theorem 2.2. Let a complex valued function $f(x) \in h(0, \infty)$ be given on $[0,+\infty)$. Then the solution $\varphi(x)$ of $(1.1)$ in the class $h(0, \infty)$ satisfying the condition

$$
\frac{1}{\sqrt{a^{2}-b^{2}}} \frac{1}{\pi i} \int_{0}^{+\infty} \frac{1}{\sigma^{\beta}} \frac{f(\sigma)}{\sigma+1} d \sigma=\frac{1}{\pi i} \int_{0}^{+\infty} \frac{\varphi(\sigma)}{\sigma+1} d \sigma,
$$

is given by the formula

$$
\varphi(x)=\frac{a f(x)}{a^{2}-b^{2}}-\frac{x^{\beta}}{\pi i} \int_{0}^{+\infty} \frac{1}{\sigma^{\beta}} \frac{b}{\sigma-x} f(\sigma) d \sigma, \quad x \in(0,+\infty),
$$

where $\operatorname{Re} \alpha>0, \operatorname{Re} \beta>0,0<\theta<\pi, \lim _{x \rightarrow+\infty} f(x)=0$.

Now we introduce one more formula, which will be used later to obtain an approximate solution of (2.15).

Let $P_{k}^{(\alpha, \beta)}(x)$ be a Jacobi polynomial of degree $k$ with a weight function $p(x)=(1-x)^{\alpha} \times$ $(1+x)^{\beta}, \alpha, \beta>-1$. Then it follows from [3] that

$$
\begin{gathered}
A Z(x) P_{k}^{(\alpha, \beta)}(x)+\frac{1}{\pi i} \int_{-1}^{1} B Z(t) \frac{P_{k}^{(\alpha, \beta)}(t)}{t-x} d t-\frac{1}{\pi i} \int_{-1}^{1} B Z(t) \frac{P_{k}^{(\alpha, \beta)}(t)}{t-1} d t= \\
\begin{cases}P_{k}^{(-\alpha,-\beta)}(x)-P_{k}^{(-\alpha,-\beta)}(1), & \alpha+\beta=0, \quad \kappa=0, \quad k=0,1,2, \ldots, \\
2\left[P_{k+1}^{(-\alpha,-\beta)}(x)-P_{k+1}^{(-\alpha,-\beta)}(1)\right], & \alpha+\beta=1, \quad \kappa=-1, \quad k=0,1,2, \ldots, \\
{\left[P_{k-1}^{(-\alpha,-\beta)}(x)-P_{k-1}^{(-\alpha,-\beta)}(1)\right] / 2,} & \alpha+\beta=-1, \quad \kappa=1, \quad k=1,2,3, \ldots\end{cases}
\end{gathered}
$$

\section{Approximate solution}

In this section, we will derive an approximate solution of (2.15). For this purpose, we will interpolate the function $g(t)$ at Chebyshev nodes

$$
t_{k}=\cos \frac{(2 k-1) \pi}{2(n+1)}, \quad k=1,2, \ldots, n+1,
$$

by a polynomial $g_{n}(t)$ of degree $n$. We will use the following approximation formula (cf. [7])

$$
g_{n}(t)=\frac{2}{n+1} \sum_{j=0}^{n}\left(\sum_{k=1}^{n+1} T_{j}\left(t_{k}\right) g\left(t_{k}\right)\right) T_{j}(t),
$$


where $T_{j}(t)=\cos (j \arccos t)$ are Chebyshev polynomials of the first kind. Expressing Chebyshev polynomials $T_{j}(t)$ in terms of Jacobi polynomials, we obtain

$$
T_{j}(t)=\sum_{l=0}^{j} \rho_{j l} P_{l}^{(-\alpha,-\beta)}(t)
$$

where

$$
\begin{gathered}
\rho_{j l}=\frac{1}{h_{l}^{(-\alpha,-\beta)}} \frac{1}{\pi} \int_{-1}^{1} q(t) T_{j}(t) P_{l}^{(-\alpha,-\beta)}(t) d t= \\
(-1) \frac{1}{h_{l}^{(-\alpha,-\beta)}} \frac{1}{\sin \pi \alpha} \operatorname{Res}_{z=\infty}\left\{(z-1)^{-\alpha}(z+1)^{-\beta} T_{j}(z) P_{l}^{(-\alpha,-\beta)}(z)\right\} \\
q(t)=(1-t)^{-\alpha}(1+t)^{-\beta}, \quad \alpha+\beta=0, \\
h_{l}^{(-\alpha,-\beta)}=\frac{1}{\pi} \int_{-1}^{1} q(t)\left[P_{l}^{(-\alpha,-\beta)}(t)\right]^{2} d t=\frac{2}{(2 l+1) \pi} \frac{\Gamma(l-\alpha+1) \Gamma(l-\beta+1)}{l ! \Gamma(l+1)} .
\end{gathered}
$$

Using (3.3), the interpolation polynomial (3.2) takes the form

$$
g_{n}(t)=\sum_{k=0}^{n} g_{k} P_{k}^{(-\alpha,-\beta)}(t)
$$

where

$$
g_{0}=\frac{2}{n+1} \sum_{j=0}^{n} '\left(\sum_{i=1}^{n+1} T_{j}\left(t_{i}\right) g\left(t_{i}\right)\right) \rho_{j 0}, \quad g_{k}=\frac{2}{n+1} \sum_{j=k}^{n}\left(\sum_{i=1}^{n+1} T_{j}\left(t_{i}\right) g\left(t_{i}\right)\right) \rho_{j k}, \quad k=1,2, \ldots, n .
$$

Following [11], an approximate solution $u_{n}(x)$ of problem $(2.15),(2.18)$ is defined as a solution of the following problem:

$$
\begin{gathered}
A Z(t) u_{n}(t)+\frac{1}{\pi i} \int_{-1}^{1} B Z(\tau) \frac{u_{n}(\tau)}{\tau-t} d \tau-\frac{1}{\pi i} \int_{-1}^{1} B Z(\tau) \frac{u_{n}(\tau)}{\tau-1} d \tau=g_{n}(t)-g_{n}(1), \quad t \in(-1,1) \\
\frac{1}{\pi i} \int_{-1}^{1} B Z(\tau) \frac{u_{n}(\tau)}{\tau-1} d \tau=A_{0}^{*}
\end{gathered}
$$

where

$$
u_{n}(t)=\sum_{k=0}^{n} c_{k} P_{k}^{(\alpha, \beta)}(t)
$$

with unknown coefficients $c_{k}$.

Substituting (3.8) into (3.6), (3.7) and using formula (2.25) for the computation of the singular integral we obtain

$$
\sum_{k=0}^{n} c_{k}\left[P_{k}^{(-\alpha,-\beta)}(t)-P_{k}^{(-\alpha,-\beta)}(1)\right]=\sum_{k=0}^{n} g_{k}\left[P_{k}^{(-\alpha,-\beta)}(t)-P_{k}^{(-\alpha,-\beta)}(1)\right]
$$




$$
\sum_{k=0}^{n} c_{k} \frac{1}{\pi i} \int_{-1}^{1} B Z(\tau) \frac{P_{k}^{(\alpha, \beta)}(\tau)}{\tau-1} d \tau=\sum_{k=0}^{n} c_{k} P_{k}^{(-\alpha,-\beta)}(1)=A_{0}^{*}
$$

Hence, we get

$$
c_{n}=g_{n}, \ldots, c_{1}=g_{1}, \quad c_{0}=A_{0}^{*}-c_{1} P_{1}^{(-\alpha,-\beta)}(1)-\ldots-c_{n} P_{n}^{(-\alpha,-\beta)}(1) .
$$

If $\kappa=-1$, then the approximate solution of $(2.15)$

$$
u_{n-1}(t)=\sum_{k=0}^{n-1} c_{k} P_{k}^{(\alpha, \beta)}(t),
$$

where $c_{k}$ are the coefficients to be determined, can be found as a solution of the following problem:

$A Z(t) u_{n-1}(t)+\frac{1}{\pi i} \int_{-1}^{1} B Z(\tau) \frac{u_{n-1}(\tau)}{\tau-t} d \tau-\frac{1}{\pi i} \int_{-1}^{1} B Z(\tau) \frac{u_{n-1}(\tau)}{\tau-1} d \tau=g_{n}(t)-g_{n}(1), \quad t \in(-1,1)$,

with $g_{n}(t)=\sum_{k=0}^{n} g_{k} P_{k}^{(-\alpha,-\beta)}(t)$, where

$$
g_{0}=\frac{2}{n+1} \sum_{j=0}^{n} '\left(\sum_{i=1}^{n+1} T_{j}\left(t_{i}\right) g\left(t_{i}\right)\right) \rho_{j 0}, \quad g_{k}=\frac{2}{n+1} \sum_{j=k}^{n}\left(\sum_{i=1}^{n+1} T_{j}\left(t_{i}\right) g\left(t_{i}\right)\right) \rho_{j k}, \quad k=1,2, \ldots, n,
$$

and

$$
\begin{gathered}
\rho_{j k}=\frac{1}{h_{k}^{(-\alpha,-\beta)}} \frac{1}{\pi} \int_{-1}^{1} q(t) T_{j}(t) P_{k}^{(-\alpha,-\beta)}(t) d t= \\
(-1) \frac{1}{h_{k}^{(-\alpha,-\beta)}} \frac{1}{\sin \pi \alpha} \operatorname{Res}_{z=\infty}\left\{(z-1)^{-\alpha}(z+1)^{-\beta} T_{j}(z) P_{k}^{(-\alpha,-\beta)}(z)\right\}, \\
q(t)=(1-t)^{-\alpha}(1+t)^{-\beta}, \quad \alpha+\beta=1, \\
h_{k}^{(-\alpha,-\beta)}=\frac{1}{\pi} \int_{-1}^{1} q(t)\left[P_{k}^{(-\alpha,-\beta)}(t)\right]^{2} d t=\frac{2}{(k+1) \pi} \frac{\Gamma(k-\alpha+1) \Gamma(k-\beta+1)}{k ! \Gamma(k+2)} .
\end{gathered}
$$

Using (2.22) formula (3.10) can be rewritten in the form

$$
\begin{gathered}
A Z(t) u_{n-1}(t)+\frac{1}{\pi i} \int_{-1}^{1} B Z(\tau) \frac{u_{n-1}(\tau)}{\tau-t} d \tau=g_{n}(t)+\frac{1}{\pi i} \int_{-1}^{1} b Z^{-1}(\tau) g_{n}(\tau) d \tau= \\
g_{n}(t)-g_{0}=\sum_{k=1}^{n} g_{k} P_{k}^{(-\alpha,-\beta)}(t), \quad t \in(-1,1) .
\end{gathered}
$$

Substituting (3.9) into (3.11) and using formula (2.25), we obtain

$$
2 \sum_{k=0}^{n-1} c_{k} P_{k+1}^{(-\alpha,-\beta)}(t)=\sum_{k=1}^{n} g_{k} P_{k}^{(-\alpha,-\beta)}(t)
$$

Hence, we finally obtain

$$
c_{n-1}=\frac{1}{2} g_{n}, \quad c_{n-2}=\frac{1}{2} g_{n-1}, \quad \ldots, \quad c_{0}=\frac{1}{2} g_{1} .
$$




\section{Estimation of errors}

In this section, we will estimate the orders of errors of approximate solutions.

Theorem 4.1. Let the right-hand side $g(t)$ of (2.15) satisfy the Hölder condition

$$
\left|g\left(t^{\prime}\right)-g\left(t^{\prime \prime}\right)\right| \leqslant K\left|t^{\prime}-t^{\prime \prime}\right|^{\mu}, \quad-1<t^{\prime}, t^{\prime \prime}<1, \quad 0<\mu \leqslant 1 .
$$

Moreover, let $g(t)$ be approximated by the interpolation polynomial (3.4) with respect to the Chebyshev nodes of the first kind (3.1), and let $u(t)$ and $u_{n}(t)$ denote the exact and approximate solutions of the problem given by (2.15), (2.18) and (3.6), (3.10). Then the following estimation holds:

$$
\left\|(1-t)^{\alpha}\left(u(t)-u_{n}(t)\right)\right\|_{\infty} \leqslant M n^{-\mu} \ln ^{2} n
$$

where $\alpha$ is given by (2.7) and (2.9) and $M$ is a constant not depending on $n$.

Proof. Since

$$
u_{n}(t)=a Z^{-1}(t) g_{n}(t)-\frac{1}{\pi i} \int_{-1}^{1} b Z^{-1}(\tau) \frac{g_{n}(\tau)}{\tau-t} d \tau+\gamma_{0}, \quad-1<t<1,
$$

we have

$$
u(t)-u_{n}(t)=a Z^{-1}(t)\left[g(t)-g_{n}(t)\right]-\frac{1}{\pi i} \int_{-1}^{1} b Z^{-1}(\tau) \frac{g(\tau)-g_{n}(\tau)}{\tau-t} d \tau, \quad-1<t<1 .
$$

In this case, the integral on the right-hand side of (4.3) can have an integrable singularity at the point $t=1$. Therefore, we have to estimate the product

$$
(1-t)^{\alpha} \frac{1}{\pi i} \int_{-1}^{1} b Z^{-1}(\tau) \frac{g(\tau)-g_{n}(\tau)}{\tau-t} d \tau, \quad-1<t<1,
$$

for which we have the following estimation [11]:

$$
\left\|(1-t)^{\alpha} \frac{1}{\pi i} \int_{-1}^{1} b Z^{-1}(\tau) \frac{g(\tau)-g_{n}(\tau)}{\tau-t} d \tau\right\| \leqslant M_{1} \frac{\ln ^{2} n}{n^{\mu}},
$$

where $M_{1}$ is a constant not depending on $n$. Taking into account inequality [7]

$$
\left\|g(t)-g_{n}(t)\right\|_{\infty} \leqslant M_{2} n^{-\mu} \ln n
$$

where $M_{2}$ is a constant not depending on $n$, we obtain result (4.2).

Theorem 4.2. Suppose that the conditions of the Theorem 4.1 are satisfied (i.e., the right-hand side function $g(t)$ and its approximation $g_{n}(t)$ are the same as in Theorem 4.1), and let $u(t), u_{n-1}(t)$ denote the exact and approximate solutions of the problem defined by (2.15), (3.10) respectively. Then the following estimate holds:

$$
\left\|Z(t)\left[u(t)-u_{n-1}(t)\right]\right\|_{\infty} \leqslant M n^{-\mu} \ln ^{2} n,
$$

where $M$ is a constant not depending on $n$. 
In the last case, the integral on the right-hand side of (4.3) can have an integrable singularity at the endpoints of the interval $[-1,1]$. Therefore, we have to estimate the product

$$
Z(t) \frac{1}{\pi i} \int_{-1}^{1} b Z^{-1}(\tau) \frac{g(\tau)-g_{n}(\tau)}{\tau-t} d \tau, \quad-1<t<1,
$$

for which we have the following estimation [11]:

$$
\left\|Z(t) \frac{1}{\pi i} \int_{-1}^{1} b Z^{-1}(\tau) \frac{g(\tau)-g_{n}(\tau)}{\tau-t} d \tau\right\|_{\infty} \leqslant M_{1} \frac{\ln ^{2} n}{n^{\mu}}
$$

where $M_{1}$ is a constant not depending on $n$.

Remark 4.1. If $g(t), t \in(-1,1)$, being the right-hand side of (2.15), satisfies the Hölder condition (4.1), then the function $f(x), x>0$, being the right-hand side of (1.1) also satisfies the Hölder inequality.

\section{Numerical example}

In this section we will present a numerical example which illustrates usefulness of the approximation algorithms presented in the previous sections.

Let us consider the characteristic equation (1.1) in the class $h(0, \infty)(\kappa=-1)$, where

$$
a=1+\frac{i}{2}, \quad b=-\frac{i}{2}, \quad f(x)=\frac{1}{2 x+3} .
$$

Then

$$
g(t)=f\left(\frac{1+t}{1-t}\right)=\frac{t-1}{t-5} .
$$

It is easy to check that the exact solution of $(2.15)$ in the class $h(-1,1)(\kappa=-1)$ is equal to

$$
u(t)=\frac{4}{X(5)(t-5)}
$$

The values of the exact and approximated solutions $u_{n-1}(x)$ of $(2.15)$ for $n=10$ are shown in Table.

Comparison of the values of the exact and approximated solutions of (2.15)

\begin{tabular}{|c|c|c|c|}
\hline$x$ & $t$ & $u(t)$ & $u_{n}(t)$ \\
\hline 0,11 & $-0,8$ & $-0,1208880939359+0,002704114228534 i$ & $-0,1208880939315+0,002704114229119 i$ \\
0,25 & $-0,6$ & $-0,1252055258622+0,002800689736696 i$ & $-0,1252055258366+0,002800689749419 i$ \\
0,538 & $-0,3$ & $-0,1322926310997+0,002959219344433 i$ & $-0,1322926310882+0,002959219344380 i$ \\
1 & 0 & $-0,1402301889657+0,003136772505099 i$ & $-0,1402301889676+0,003136772510523 i$ \\
1,5 & 0,2 & $-0,1460731135059+0,003267471359479 i$ & $-0,1460731135102+0,003267471359345 i$ \\
2,333 & 0,4 & $-0,1524241184410+0,003409535331630 i$ & $-0,1524241184220+0,00340953533329 i$ \\
3 & 0,5 & $-0,1558113210730+0,00348530278344 i$ & $-0,1558113210675+0,003485302787656 i$ \\
4 & 0,6 & $-0,1593524874610+0,003564514210340 i$ & $-0,1593524874720+0,003564514214022 i$ \\
5,666 & 0,7 & $-0,1630583592624+0,003647409889651 i$ & $-0,1630583592671+0,00364740988958 i$ \\
9 & 0,8 & $-0,1669407011496+0,003734252982261 i$ & $-0,1669407011326+0,003734252980918 i$ \\
19 & 0,9 & $-0,171012425567+0,003825332323292 i$ & $-0,1710124255673+0,003825332327957 i$ \\
\hline
\end{tabular}




\section{References}

1. R. Estrada and R. P. Kanwal, Singular integral equations, Birkhäuser, Boston, 2000.

2. F. D. Gakhov, Boundary Value Problems, International Series of Monographs in Pure and Applied Mathematics, Vol. 85, Pregamon Press, Oxford, 1966.

3. P. Karczmarek, D. Pylak, and M. A. Sheshko, Application of Jacobi polynomials to approximate solution of a singular integral equation with Cauchy kernel, Applied Mathematics and Computation [in press].

4. L. N. Karpenko, Approximate solution of a singular integral equation with the use of Jacobi polynomials, Prikl. Mat. i Mekh., (1966), no. 3, pp. 564-569 (in Russian).

5. E. G. Ladopoulos, Singular Integral Equations. Linear and Non-linear Theory and its Applications in Science and Engineering, Springer-Verlang, 2000.

6. N. I. Muskhelishvilli, Singular Integral Equations, Nauka, Moscow, 1968 (in Russian).

7. S. Paszkowski, Numerical Applications of Chebyshev Polynomials and Series, PWN, Warsaw, 1975.

8. A. P. Prudnikow, Y. A. Brychkov, and O. I. Marchiev, Integrals and Series, Vol. 5, Gordon \& Breach Sci. Publ., New York, 1992.

9. D. Pylak, R. Smarzewski, and M. A. Sheshko, A Singular Integral Equation with a Cauchy Kernel on the Real Half-Line, Differential Equations, 41 (2005), no. 12, pp. 1696-1708.

10. D. Pylak and M. A. Sheshko, Inversion of Singular Integrals with Cauchy Kernels in the Case of an Infinite Integration Domain, Differential Equations, 41 (2005), no. 9, pp. 1229-1241.

11. M. Sheshko, Singular Integral Equations with Cauchy and Hilbert Kernels and Theirs Appoximated Solutions, The Learned Society of the Catholic University of Lublin, Lublin, 2003 (in Russian).

12. G. Szegö, Orthogonal polynomials, American Mathematical Society, Colloquium Publications, Vol. XXIII, 1939. 